\title{
Optical Coupling Structures of Fiber-Optic Mach-Zehnder Interferometers Using $\mathrm{CO}_{2}$ Laser Irradiation
}

\author{
Chien-Hsing Chen, ${ }^{1,2}$ Chih-Yu Hsu, ${ }^{3}$ Pei-Hsing Huang, ${ }^{4}$ Jian-Neng Wang, ${ }^{5}$ and Wei-Te Wu ${ }^{3}$ \\ ${ }^{1}$ Department of Physics, National Chung Cheng University, Chiayi 62102, Taiwan \\ ${ }^{2}$ Center for Nano Bio-Detection, National Chung Cheng University, Chiayi 62102, Taiwan \\ ${ }^{3}$ Department of Biomechatronics Engineering, National Pingtung University of Science and Technology, Pingtung 912, Taiwan \\ ${ }^{4}$ Department of Mechanical Engineering, National Pingtung University of Science and Technology, Pingtung 912, Taiwan \\ ${ }^{5}$ Department of Construction Engineering, National Yunlin University of Science and Technology, Yunlin 640, Taiwan
}

Correspondence should be addressed to Wei-Te Wu; weite@mail.npust.edu.tw

Received 3 January 2014; Accepted 3 March 2014; Published 15 June 2014

Academic Editor: Chung-Liang Chang

Copyright (c) 2014 Chien-Hsing Chen et al. This is an open access article distributed under the Creative Commons Attribution License, which permits unrestricted use, distribution, and reproduction in any medium, provided the original work is properly cited.

\begin{abstract}
The Mach-Zehnder interferometer (MZI) can be used to test changes in the refractive index of sucrose solutions at different concentrations. However, the popularity of this measurement tool is limited by its substantial size and portability. Therefore, the MZI was integrated with a small fiber-optic waveguide component to develop an interferometer with fiber-optic characteristics, specifically a fiber-optic Mach-Zehnder interferometer (FO-MZI). Optical fiber must be processed to fabricate two optical coupling structures. The two optical coupling structures are a duplicate of the beam splitter, an optical component of the interferometer. Therefore, when the sensor length and the two optical coupling structures vary, the time or path for optical transmission in the sensor changes, thereby influencing the back-end interference signals. The researchers successfully developed an asymmetrical FO-MZI with sensing abilities. The spacing value between the troughs of the sensor length and interference signal exhibited an inverse relationship. In addition, image analysis was employed to examine the size-matching relationship between various sensor lengths and the coupling and decoupling structure. Furthermore, the spectral wavelength shift results measured using a refractive index sensor indicate that FO-MZIs with a sensor length of $38 \mathrm{~mm}$ exhibited excellent sensitivity, measuring $59.7 \mathrm{~nm} / \mathrm{RIU}$.
\end{abstract}

\section{Introduction}

Recently, several types of optical waveguide sensors or optical fiber-based refractometer have been proposed, including long period fiber gratings, Fabry-Perot interferometers, and Mach-Zehnder interferometers (MZI). The operation principle of the MZI by the first coupling point induces coupling of a fraction of the incident light propagating in the core mode to the cladding mode, and the second one performs the opposite function, generating the interference of light propagating in different optical paths. The fiber-optic Mach-Zehnder interferometer (FO-MZI) is a miniaturized and economical sensor component with label-free, real-time detection abilities. The FO-MZI does not require surface modification to induce surface plasmon resonance (SPR) [1] or particle plasmon resonance (PPR) [2]. The FO-MZI has been successfully employed for measuring refractive indices and temperatures and exhibits considerable future market potential [3]. The advantage of this type of sensor is relatively simple in construction, compact, low cost, ease of use, immunity to electromagnetic interference, and high sensitivity to the external refractive index (RI).

This study proposes a coupling and decoupling structure (which is a duplicate of the beam splitter in traditional interferometers) for the FO-MZI; thus, the structural characteristics are identical to those of a beam splitter, inducing coupling and decoupling during fiber-optic transmissions [4], as shown in Figure 1. Previous studies have examined the FO-MZI; however, few have investigated the influence that sensor length $(L)$ variations have on interference signals. The optical coupling and decoupling structure does not need to be linked to an optical source or spectrometer. Interference 


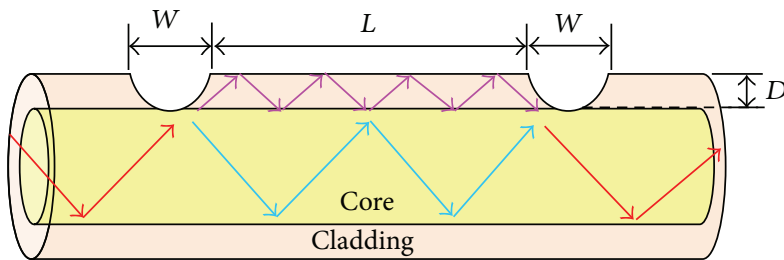

(a) The fiber-optic Mach-Zehnder interferometer

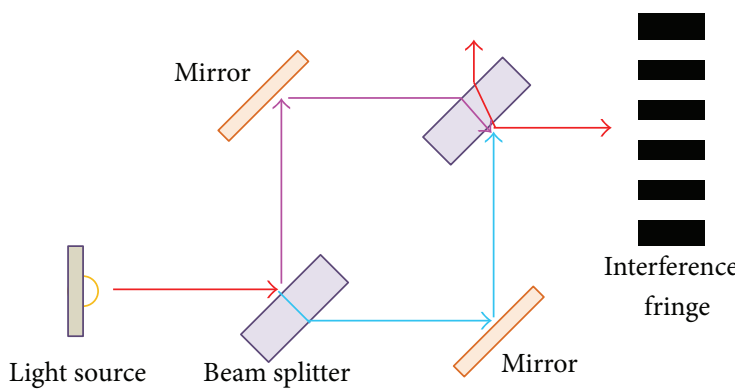

(b) The traditional Mach-Zehnder interferometer

FIGURE 1: Optical transmission path.

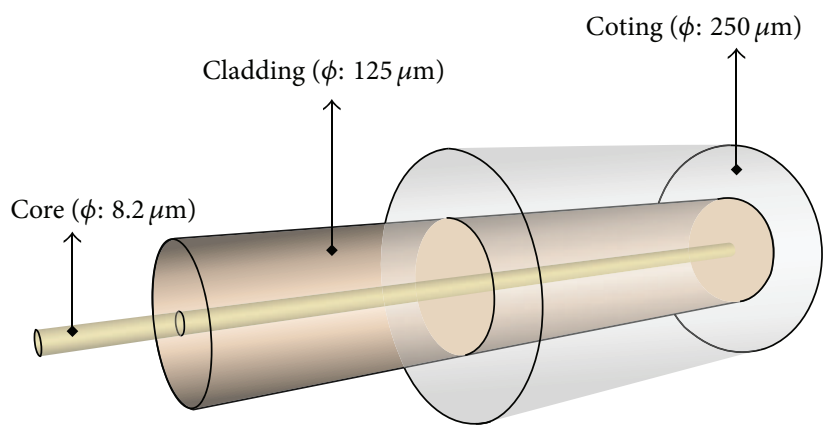

FIgURE 2: Single-mode optical fiber.

signals are obtained by satisfying the size-matching criterion for the specific structure.

\section{Experimental}

2.1. Fabrication of FO-MZI Using a $\mathrm{CO}_{2}$ Laser. This study adopted a $\mathrm{CO}_{2}$ laser-processing method, which is simple, rapid, and flexible for correcting basic parameters. We employed a single-mode communication optical fiber as the material for producing the FO-MZI because intermodal dispersion does not exist in single modes; therefore, single modes can be applied to long-distance transmissions. Furthermore, the small size of the single-mode communication optical fiber is ideal (see Figure 2) [5].

The production process equipment for the $\mathrm{CO}_{2}$ laser is shown in Figure 3. The researchers used a crimping tool to remove the fiber cladding layer covering the area required to develop the coupling and decoupling structure. The optical fiber was subsequently placed on a $\mathrm{CO}_{2}$ laser-processing platform, and an amplified spontaneous emission CL waveband optical source was steered onto the left end of the optical fiber [3]. An optical spectrum analyzer was connected to the right end of the optical fiber. Suitable processing parameters for the $\mathrm{CO}_{2}$ laser were adjusted using a computer, and a heat removal method was employed, using the $\mathrm{CO}_{2}$ laser to remove the specific target area. Thus, the coupling and decoupling structure was established, satisfying the criterion for forming interference.
2.2. Principle of FO-MZI. A schematic of FO-MZI is shown in Figure 1(a), in which sensing length $L$ denotes the distance between the two coupling points. The beam splitters of the MZI were made by forming two coupling regions at two positions separated by a distance $L$ in the fiber. An incident light propagating as a core mode is split into the core mode and the cladding modes after passing through the first coupling region. The light of the cladding modes is recombined with the core mode after the second coupling region, resulting in an interference pattern if the phase matching condition is properly fulfilled. This section of single-mode fiber with a pair of coupling regions functions as a MZI interferometer. The interference fringe pattern can be finely tuned by adjusting the loss strength of the two coupling regions and their separation.

2.3. Sensor Length Control. This study covered the following Ls for the FO-MZI: $10 \mathrm{~mm}, 20 \mathrm{~mm}, 30 \mathrm{~mm}, 40 \mathrm{~mm}$, and $50 \mathrm{~mm}$. Each length of FO-MZI samples comprised 10 sensors. Optical sources and spectrometers were used for realtime monitoring to observe the variations in interference signals for FO-MZIs with different lengths.

2.4. Measurements for the Coupling and Decoupling Structures. In this study, the FO-MZI produced using a $\mathrm{CO}_{2}$ laser was asymmetrical (see Figure 1). In other words, the coupling and decoupling structure was only on one side of the optical fiber. The removed area was small, the structure was simple, and the 


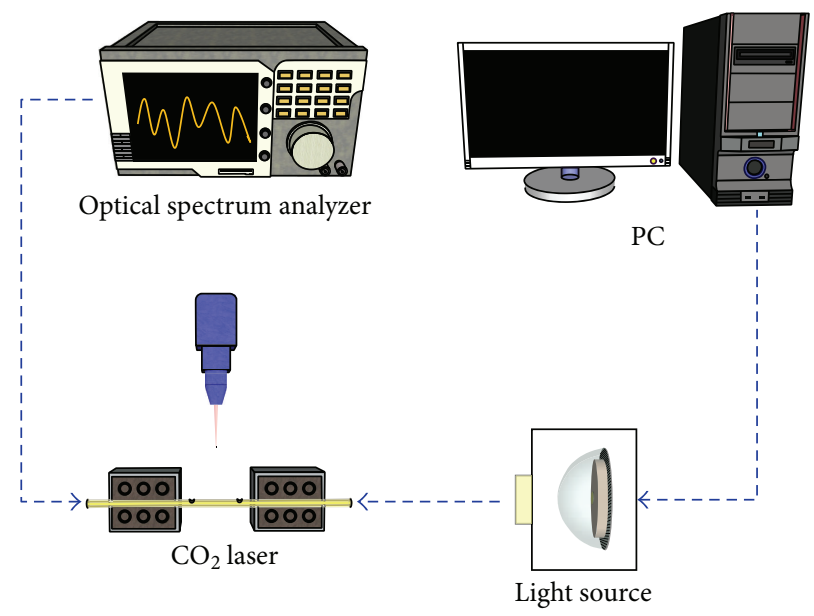

FIGURE 3: Schematic of FO-MZI production process with $\mathrm{CO}_{2}$ laser.

TABLE 1: The relationship between $L$ and interference signals.

\begin{tabular}{lccccc}
\hline \multicolumn{6}{c}{ The number of samples $=10$} \\
\hline$L(\mathrm{~mm})$ & 10 & 20 & 30 & 40 & 50 \\
Average spacing $(\mathrm{nm})$ & 45.9 & 26.6 & 16.8 & 13.8 & 9.9 \\
$=\left(\Delta \lambda_{1}+\Delta \lambda_{2}+\cdots+\Delta \lambda_{n}\right) / n$ & & & & & \\
Standard deviation $(\mathrm{nm})$ & 2.29 & 2.29 & 0.38 & 0.10 & 0.15 \\
\hline
\end{tabular}

duration was short. Therefore, the occurrence of production errors was reduced. Because of its excellent mechanical strength, the optical fiber was not prone to breaking. In addition, the radially symmetric exterior of the optical fiber was not welded [7] or etched [8].

The heat accumulation and thermal diffusion effects of the material used for $\mathrm{CO}_{2}$ laser-processing affect a portion of the material. Therefore, this section presents the use of a noncontact image-measuring instrument for measuring the size-matching relationship between FO-MZIs with different $L s$ to establish a database as a reference for future production processes. However, general detection platforms cannot be used to fixate the FO-MZI accurately, leading to measurement and determination errors, thereby reducing the reliability of the acquired size. To eliminate measurement errors, the fixture shown in Figure 4 was designed. At the center of this fixture, an auxiliary line was hypothesized. The center line divides two equal parts (see Figure 5). The optical coupling and decoupling structure was adjusted to satisfy the criterion of the center line, establish a side view of the structure, and achieve a vertical charged-coupled device lens for correction.

2.5. Refractive Index Detection. As shown in the experiment framework presented in Figure 6, we placed the FO-MZI on a detection platform and connected each end of the sensor to an optical source and spectrometer to detect variations in the interference signals. The test solution was then introduced into a butterfly needle catheter using a syringe. Deionized (DI) water with a refractive index of 1.333 RIU was injected, and sucrose solutions between 1.343 RIU and 1.373 RIU were separately injected into the microfluidic channel of the chip. The injected solutions interacted with the FO-MZI in the microfluidic channel, as shown in Figure 7. A shift in the interference signal was observed, and the FO-MZI having the optimal $L$, which exhibited excellent sensitivity, was subsequently identified.

\section{Results and Discussion}

3.1. The Relationship between Various Lengths and Interference Signal. The analysis results of the spacing value between troughs of various $L s$ are shown in Table 1 . The results in Table 1 indicated that an inverse relationship existed between $L$ and the average spacing value between troughs of various Ls. This inverse relationship results from variations in the interference signal caused by the direct influence that a specific $L$ has on the time for transmitting split beams to the coupling structure $[4,9]$. Consider

$$
\Delta \lambda \times L=\frac{\lambda_{0}^{2}}{\Delta n_{\mathrm{eff}}},
$$

where $\lambda$ represents the center wavelength and $\Delta n_{\text {eff }}=$ $\Delta n_{\text {eff }}^{\text {core }}-\Delta n_{\text {eff }}^{\text {clading }}$ indicates the effective refractive index of various modes. Both values are fixed; therefore, when the $L$ is increased, the spacing value $\Delta \lambda$ between the troughs of the interference signal decreases. This finding verified the inverse relationship between the $L$ and the spacing value between the troughs of the FO-MZI developed in this study, thereby validating the theoretical relationship. The fitting results were approximate to an inverse curve, which indicated that the basic requirements for the FO-MZI were fulfilled, as shown in Figure 8. The relationship between various $L$ s and the spacing values between troughs are shown in Figure 9. The average spacing is given by

$$
\frac{\left(\Delta \lambda_{1}+\Delta \lambda_{2}+\cdots+\Delta \lambda_{n}\right)}{n}
$$




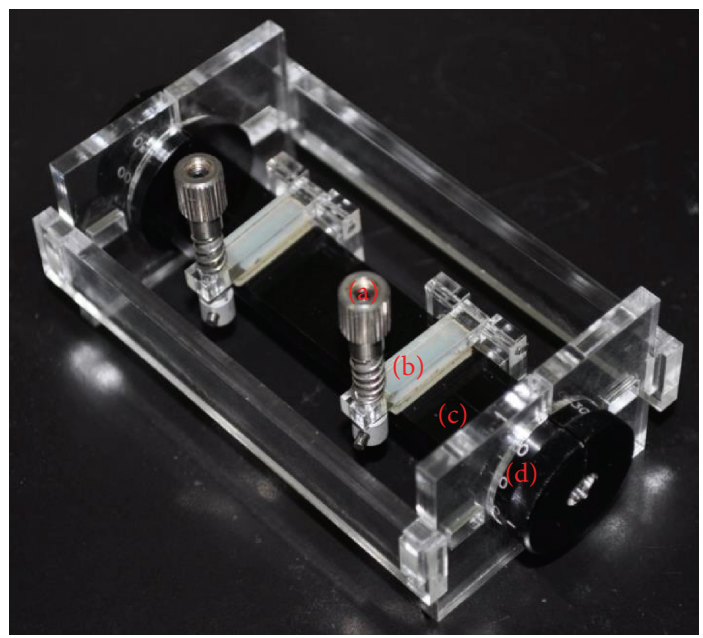

FIGURE 4: Fixture for the image-measuring instrument: (a) spring struts, (b) silicone gasket, (c) detection platform, and (d) scale turntable.

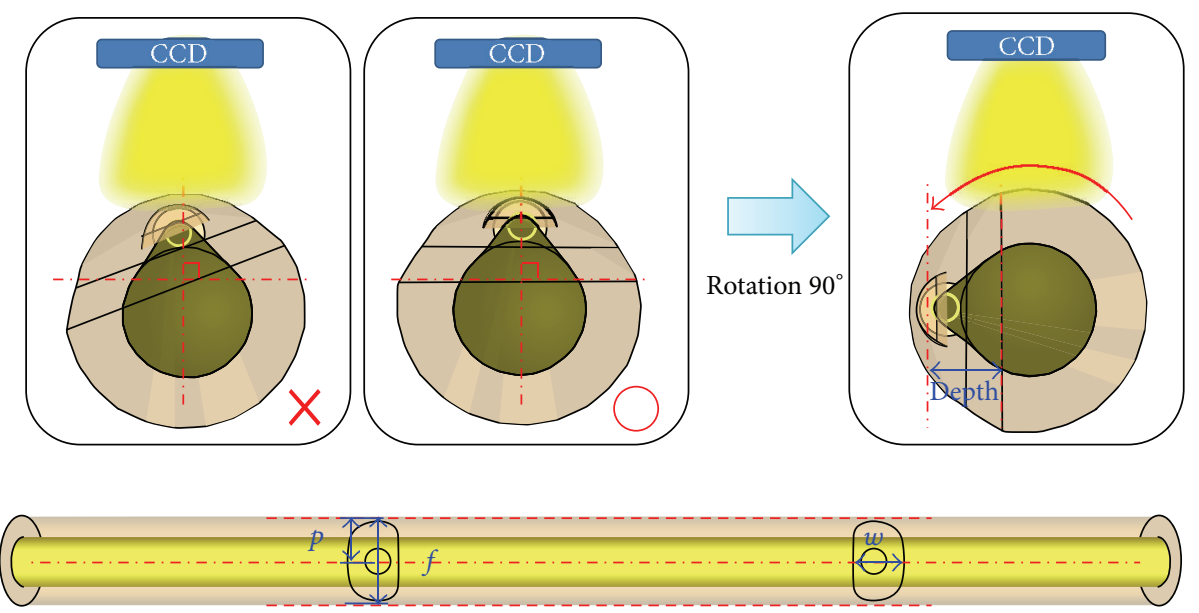

FIGURE 5: Image measurement technique.

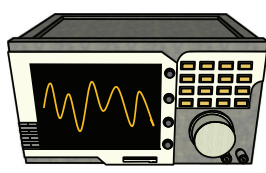

Optical spectrum analyzer
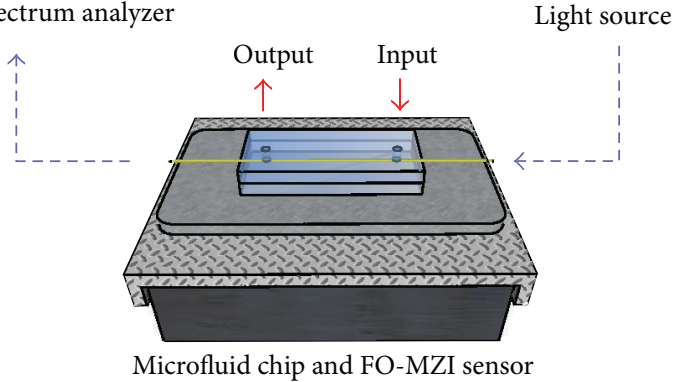

FIGURE 6: Schematic of refractive index detection using FO-MZI sensors. 
TABLE 2: The size matching of various $L s$ with the coupling and decoupling structure.

\begin{tabular}{|c|c|c|c|c|c|}
\hline \multirow{2}{*}{$L(\mathrm{~mm})$} & \multicolumn{2}{|c|}{ Width of removal $(W)(\mu \mathrm{m})$} & \multicolumn{2}{|c|}{ Depth of removal $(D)(\mu \mathrm{m})$} & \multirow{2}{*}{ Samples (sensors) } \\
\hline & Mean & Standard deviation & Mean & Standard deviation & \\
\hline 10 & 163.90 & 2.83 & 32.80 & 1.57 & \\
\hline 20 & 168.05 & 4.74 & 40.85 & 1.77 & \\
\hline 30 & 165.50 & 2.69 & 49.70 & 3.53 & 10 \\
\hline 40 & 162.30 & 4.95 & 44.35 & 4.88 & \\
\hline 50 & 163.65 & 5.30 & 37.75 & 3.89 & \\
\hline
\end{tabular}

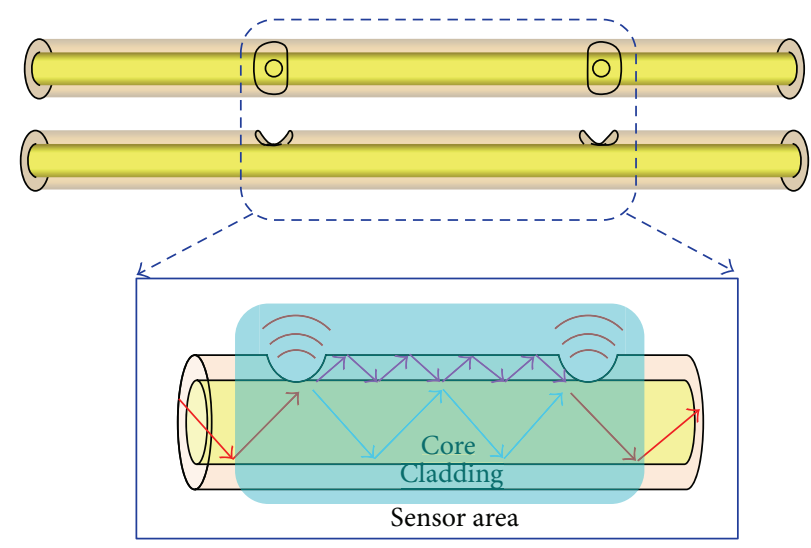

FIGURE 7: Structures of the FO-MZI sensor for refractive index detection.

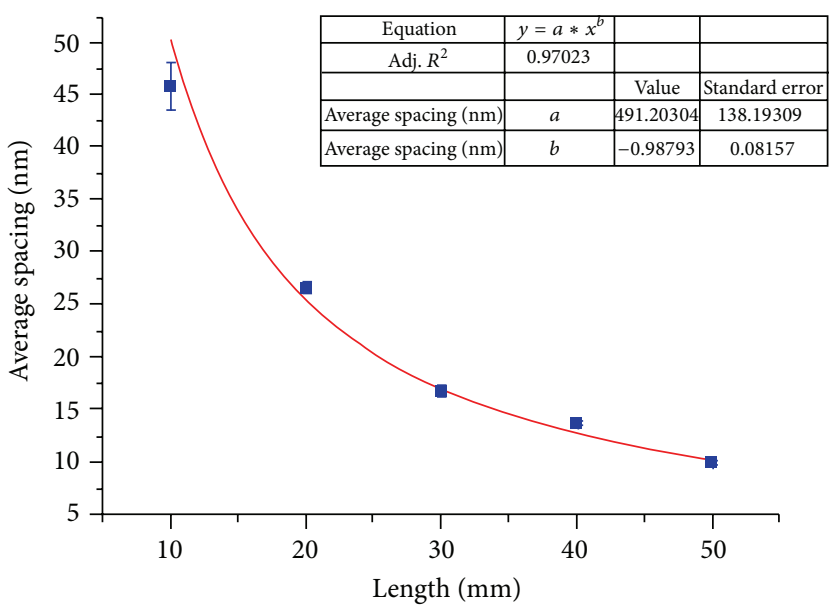

FIGURE 8: The relationship between various lengths and the spacing value between the troughs.

where $\Delta \lambda_{n}$ is the spacing value between the troughs of the interference signal and $n$ is the number of spacing.

3.2. Optical Coupling and Decoupling Structures. The image measurement results of FO-MZIs with various Ls (see Table 2) show that the size matching of the structural width and depth varied according to the $L$, as shown in Figure 10. When the $\mathrm{CO}_{2}$ laser was used to produce a coupling and decoupling structure, an FO-MZI with interference signals can be established after satisfying the matching relationship criterion of the specific database. However, repeated processing resulted in greater removal of the material for the coupling and decoupling structure, directly affecting the depth of the structure.

3.3. Refractive Index Measurement Results for Various Lengths. The experiments were repeated three times to measure the refractive index of various $L s$ according to spectral signal variations, as shown in Table 3. This study showed that the fluidic channel of general sensor chips is linear, whereas the FO-MZI is asymmetrical, as shown in Figure 11. Therefore, ensuring that the chip is placed precisely on the coupling and decoupling structure can be difficult during packaging. Other factors, such as the phase angle and test solution residue, can also influence the signal detection stability. 


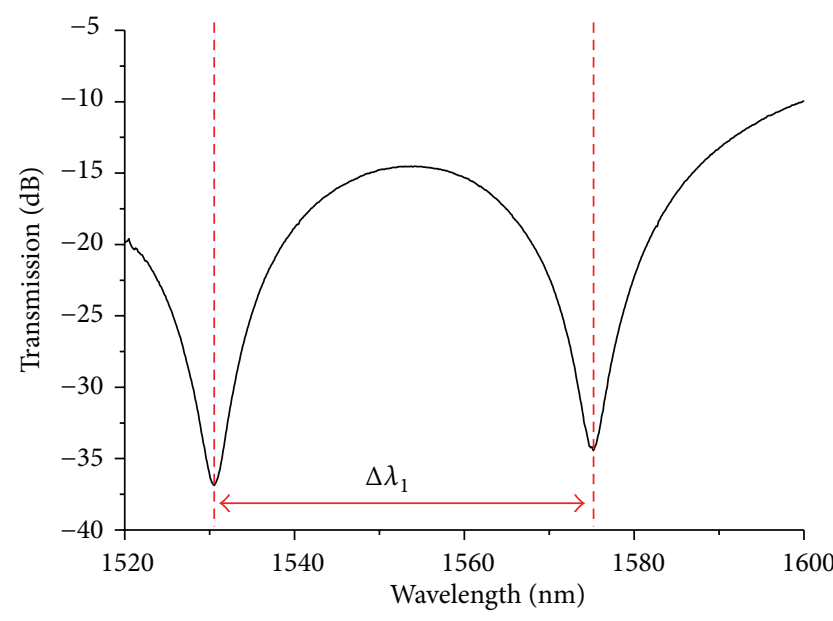

— Length $10 \mathrm{~mm}$

(a)

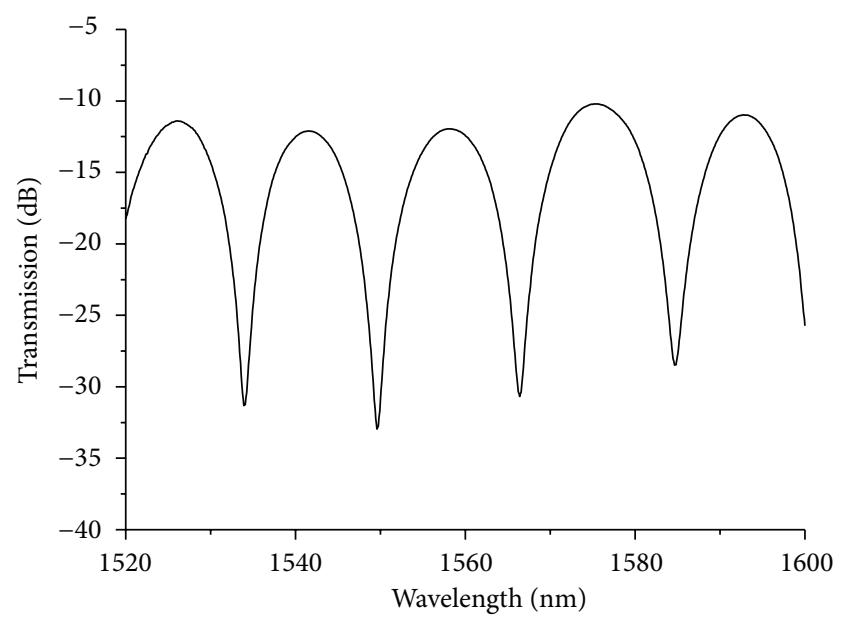

Length $30 \mathrm{~mm}$

(c)

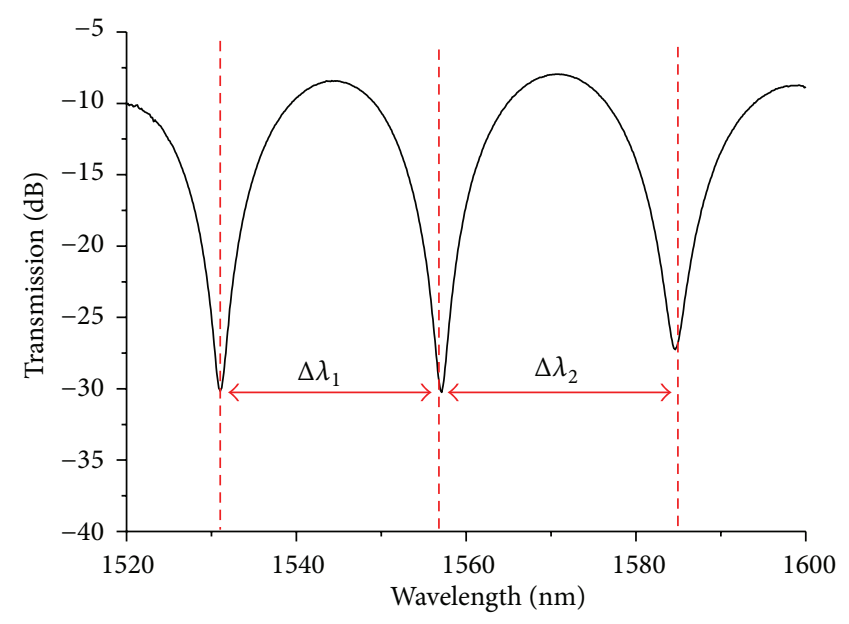

(b)

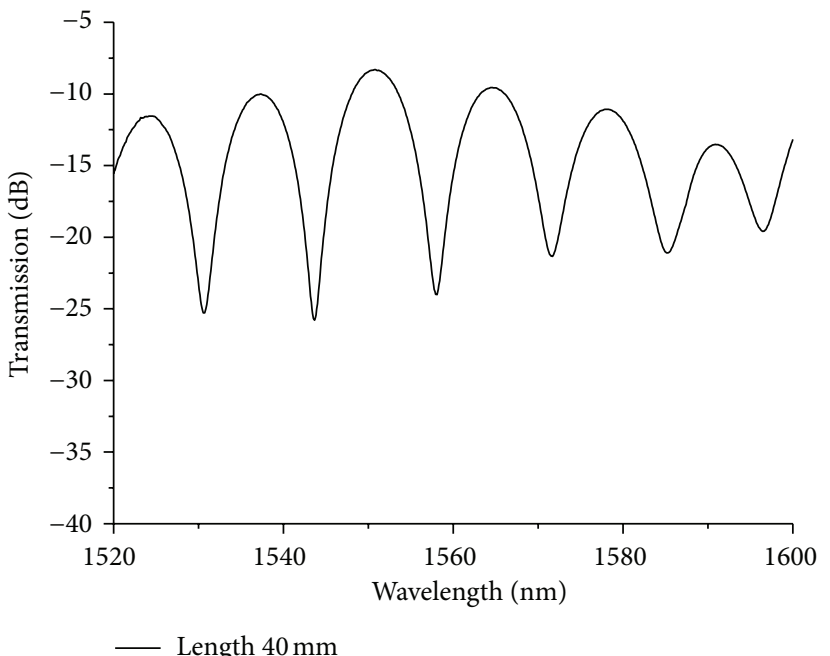

(d)

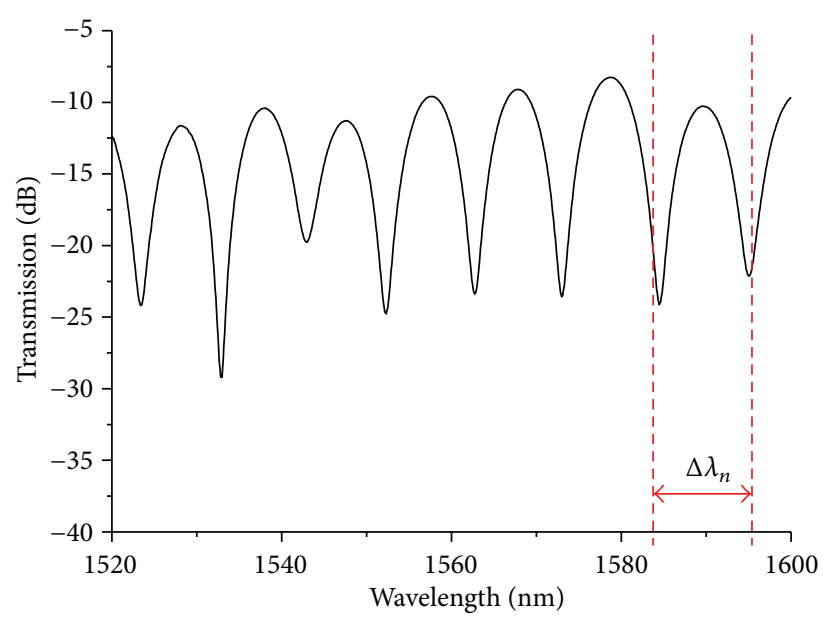

— Length $50 \mathrm{~mm}$

(e)

FigurE 9: The relationship between various lengths and interference signals. 


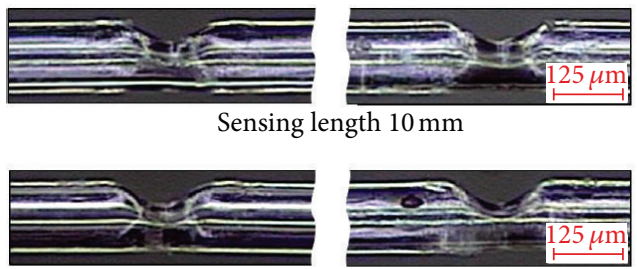

Sensing length $20 \mathrm{~mm}$
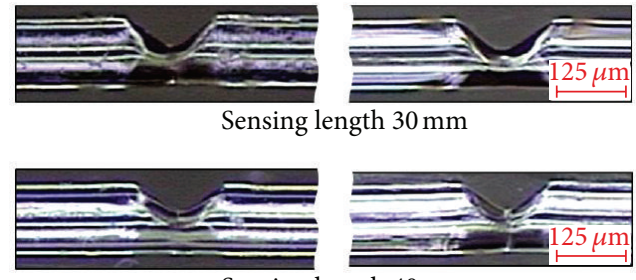

Sensing length $40 \mathrm{~mm}$

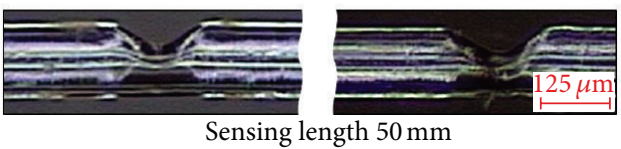

Figure 10: The image measurement results of various lengths.

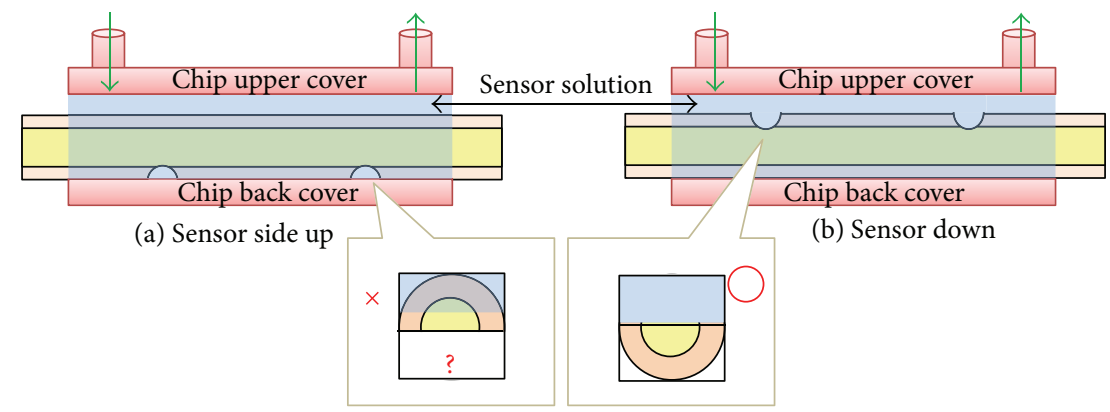

FIGURE 11: Influence of the linear fluidic channel chip.
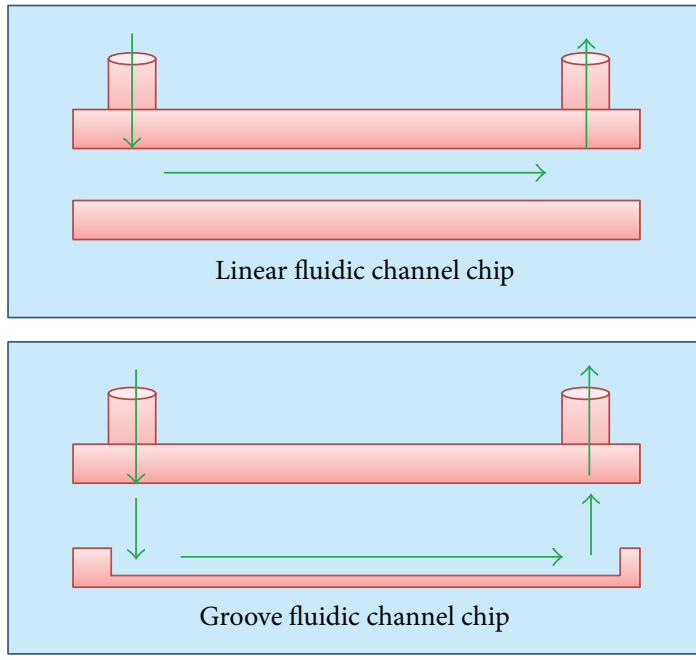

FIGURE 12: Linear and groove fluidic channel chips. 


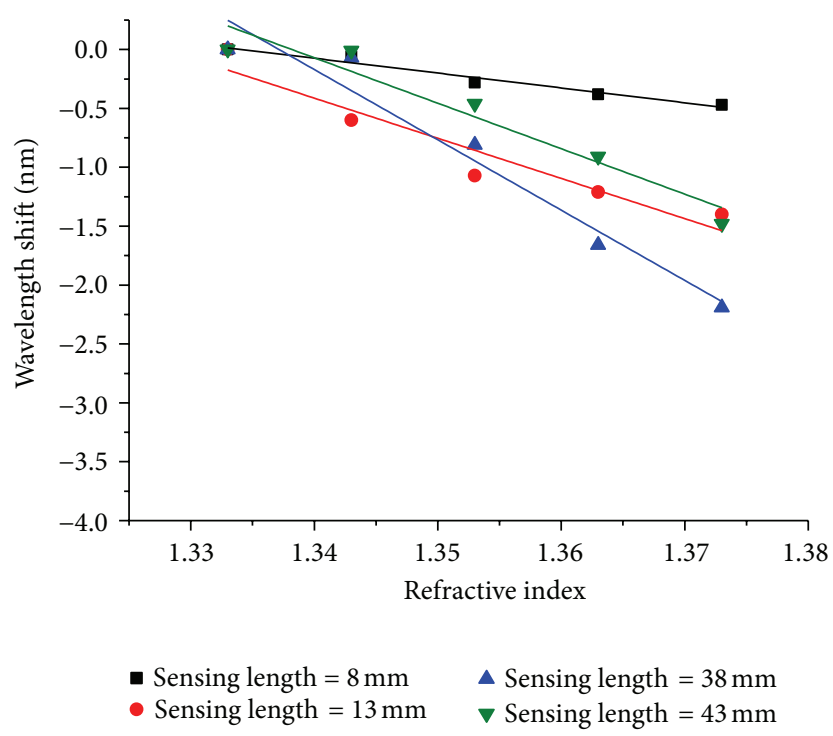

Figure 13: Refractive index measurement results for the reduced $L$ range.

TABLE 3: Refractive index measurement for various lengths (groove type).

\begin{tabular}{lccccc}
\hline $\begin{array}{l}\text { Length } \\
(\mathrm{mm})\end{array}$ & $\begin{array}{c}\text { Number } \\
01\end{array}$ & $\begin{array}{c}\text { Number } \\
\text { (nm/RIU) }\end{array}$ & $\begin{array}{c}\text { Number } \\
\text { 03 }\end{array}$ & $\begin{array}{c}\text { Average } \\
(\mathrm{nm} / \mathrm{RIU})\end{array}$ & $\begin{array}{c}\text { Standard } \\
\text { deviation } \\
(\mathrm{nm} / \mathrm{RIU})\end{array}$ \\
\hline 10 & 17.3 & 16.3 & 17.0 & 16.87 & 0.51 \\
20 & 12.2 & 9.6 & 10.4 & 10.73 & 1.33 \\
30 & 19.5 & 19.5 & 15.6 & 18.20 & 2.25 \\
40 & 54.4 & 54.4 & 54.4 & 54.40 & 0.00 \\
50 & 38.4 & 38.2 & 38.4 & 38.33 & 0.11 \\
\hline
\end{tabular}

TABLE 4: Influence of the fluidic channel shape.

\begin{tabular}{|c|c|c|c|c|}
\hline \multirow{2}{*}{$\begin{array}{l}\text { Length } \\
(\mathrm{mm})\end{array}$} & \multicolumn{2}{|c|}{$\begin{array}{l}\text { Groove fluidic } \\
\text { channel chip }\end{array}$} & \multicolumn{2}{|c|}{$\begin{array}{l}\text { Linear fluidic } \\
\text { channel chip }\end{array}$} \\
\hline & $\begin{array}{c}\text { Slope } \\
\text { (nm/RIU) }\end{array}$ & $R$-square & $\begin{array}{c}\text { Slope } \\
\text { (nm/RIU) }\end{array}$ & $R$-square \\
\hline 10 & 17.3 & 0.98856 & 30.4 & 0.96057 \\
\hline 20 & 12.2 & 0.99964 & 7.5 & 0.95356 \\
\hline 30 & 19.5 & 0.97627 & 20.8 & 0.97674 \\
\hline 40 & 54.4 & 0.97812 & 45.0 & 0.92771 \\
\hline 50 & 38.4 & 0.97728 & 20.8 & 0.97674 \\
\hline
\end{tabular}

In response to this concern, this study proposed an improved groove chip, as shown in Figure 12. Refractive index measurements of the linear and groove fluidic channel chips were obtained. The measurement results are shown in Table 4. Compared with the linear fluidic channel, the groove fluidic channel exhibited a higher linear regression value, indicating greater reliability. Furthermore, the sensing sensitivity of the groove fluidic channel chip rose from $45 \mathrm{~nm} / \mathrm{RIU}$ to $54.4 \mathrm{~nm} / \mathrm{RIU}$. Therefore, the microfluidic channel of a sensor chips is another key factor that influences the sensing results. Subsequently, the $L$ range decreased, as shown in Table 5,
TABLE 5: Refractive index measurement results for the reduced $L$ range.

\begin{tabular}{lcc}
\hline Length $(\mathrm{mm})$ & Slope $(\mathrm{nm} / \mathrm{RIU})$ & $R$-square \\
\hline 8 & 12.6 & 0.95677 \\
13 & 34.1 & 0.89198 \\
38 & 59.7 & 0.93973 \\
43 & 38.6 & 0.91275 \\
\hline
\end{tabular}

TABLE 6: The sensitivity of various optical fiber interferometers.

\begin{tabular}{lcc}
\hline $\begin{array}{l}\text { Optical fiber } \\
\text { interferometer type }\end{array}$ & $\begin{array}{c}\text { Refractive index } \\
\text { (RIU) }\end{array}$ & $\begin{array}{c}\text { Optimal sensitivity } \\
\text { value } \\
\text { (nm/RIU) }\end{array}$ \\
\hline Symmetrical [6] & $1.333 \sim 1.361$ & 26.27 \\
Fabry-Perot [7] & $1.315 \sim 1.362$ & 29.00 \\
Mismatch welding [7] & $1.315 \sim 1.362$ & 33.30 \\
Asymmetrical & $1.333 \sim 1.373$ & 59.70 \\
\hline
\end{tabular}

and an optimal sensitivity of $59.7 \mathrm{~nm} / \mathrm{RIU}$ was detected at an $L$ of $38 \mathrm{~mm}$, as shown in Figure 13. Based on the results, groove or linear fluidic channel chip (shape), sensing length, and depth of removal are important fabrication parameters of the FO-MZI sensor. The sensor sensitivity of FO-MZI can be enhanced by these parameters which is based on the evanescent wave extends $[10,11]$.

\section{Conclusion}

This study employed a $\mathrm{CO}_{2}$ laser and successfully developed an asymmetric FO-MZI with a sensing function. The sensor length $L$ between the two removals on the fiber surface and the spacing value between the troughs of the interference signal exhibited an inverse relationship. An image analysis method was proposed to examine the relationship between 
various Ls and the size matching of the coupling and decoupling structures. For future production of the FOMZI using $\mathrm{CO}_{2}$ lasers, coupling and decoupling structures with interference can be established by referencing the database proposed in this study. Finally, the results of the refractive index measurements for the spectral wavelength shifts of the FO-MZIs with various $L$ s indicate that a superior sensitivity $(59.7 \mathrm{~nm} / \mathrm{RIU})$ was exhibited at an $L$ of $38 \mathrm{~mm}$. The asymmetrical FO-MZI proposed in this study had significant advantages, including a simple structure and an easy production process. Among all interferometer types (with a coupling or decoupling structure), the refractive index measurement results of the FO-MZI exhibited higher sensitivity, as shown in Table 6. Proper selections of groove or linear fluidic channel chip (shape), sensing length, and depth of removal could yield even higher sensitivity. The unique sensing features are particularly suited for a wide variety of applications in smart structures, gain equalizer, telecommunications, and optical-sensor systems.

\section{Conflict of Interests}

The authors declare that there is no conflict of interests regarding the publication of this paper.

\section{Acknowledgments}

The support for this study provided by the National Science Council (Taiwan) through Grant nos. NSC-101-2120-M-194001-CC2, NSC-101-2221-E-020-010-MY3, NSC-102-2221-E224-065-MY2, NSC-102-2221-E-020-020, and NSC 103-2811M-194-001 is acknowledged. Chien Hsing Chen acknowledges the support of postdoctoral research fellowship from National Science Council, Taiwan.

\section{References}

[1] J. Homola, S. S. Yee, and G. Gauglitz, "Surface plasmon resonance sensors: review," Sensors and Actuators B: Chemical, vol. 54, no. 1, pp. 3-15, 1999.

[2] L.-K. Chau, Y.-F. Lin, S.-F. Cheng, and T.-J. Lin, "Fiber-optic chemical and biochemical probes based on localized surface plasmon resonance," Sensors and Actuators B: Chemical, vol. 113, no. 1, pp. 100-105, 2006.

[3] P. Lu, L. Men, K. Sooley, and Q. Chen, “Tapered fiber MachZehnder interferometer for simultaneous measurement of refractive index and temperature," Applied Physics Letters, vol. 94, no. 13, Article ID 131110, 2009.

[4] J. Hecht, Understanding Fiber Optics, Pearson, 5th edition, 2006.

[5] C. N. Chen, Fiber Optical Communications and Applications, New Wcdp, 2004.

[6] B. Li, L. Jiang, S. Wang, L. Zhou, H. Xiao, and T. Hai-Lung, "Ultra-abrupt tapered fiber mach-zehnder interferometer sensors," Sensors, vol. 11, no. 6, pp. 5729-5739, 2011.

[7] Z. Tian and S. S.-H. Yam, "In-line single-mode optical fiber interferometric refractive index sensors," Journal of Lightwave Technology, vol. 27, no. 13, pp. 2296-2306, 2009.

[8] W.-C. Kuo, Z.-Z. Feng, and N.-K. Chen, "Modal characteristics of excited cladding modes in abrupt-tapered Mach-Zehnder interferometers," in Proceedings of the 16th Opto-Electronics and Communications Conference (OECC '11), pp. 649-650, July 2011.

[9] H. Y. Choi, M. J. Kim, and B. H. Lee, "All-fiber Mach-Zehnder type interferometers formed in photonic crystal fiber," Optics Express, vol. 15, no. 9, pp. 5711-5720, 2007.

[10] D. Toomre and D. J. Manstein, "Lighting up the cell surface with evanescent wave microscopy," Trends in Cell Biology, vol. 11, no. 7, pp. 298-303, 2001.

[11] M. Oheim, D. Loerke, R. H. Chow, and W. Stühmer, "Evanescent-wave microscopy: a new tool to gain insight into the control of transmitter release," Philosophical Transactions of the Royal Society B: Biological Sciences, vol. 354, no. 1381, pp. 307-318, 1999. 

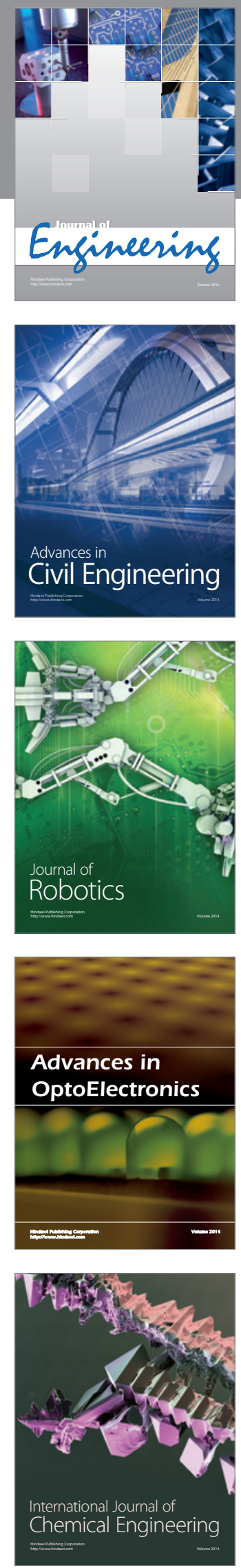

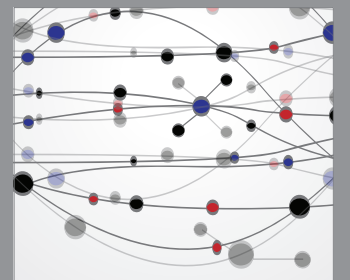

The Scientific World Journal
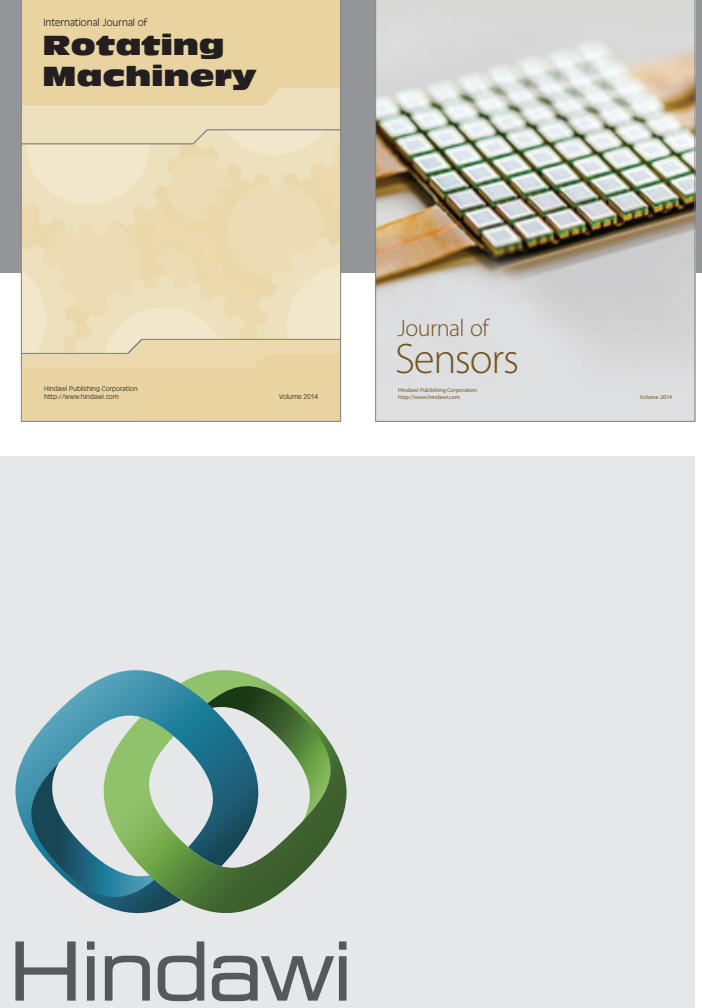

Submit your manuscripts at http://www.hindawi.com
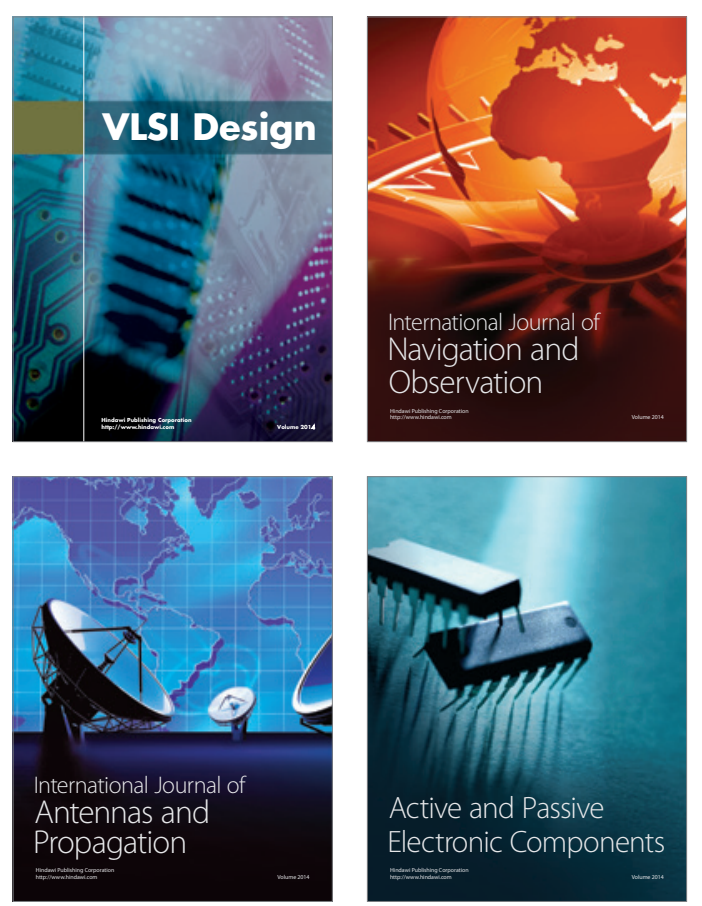
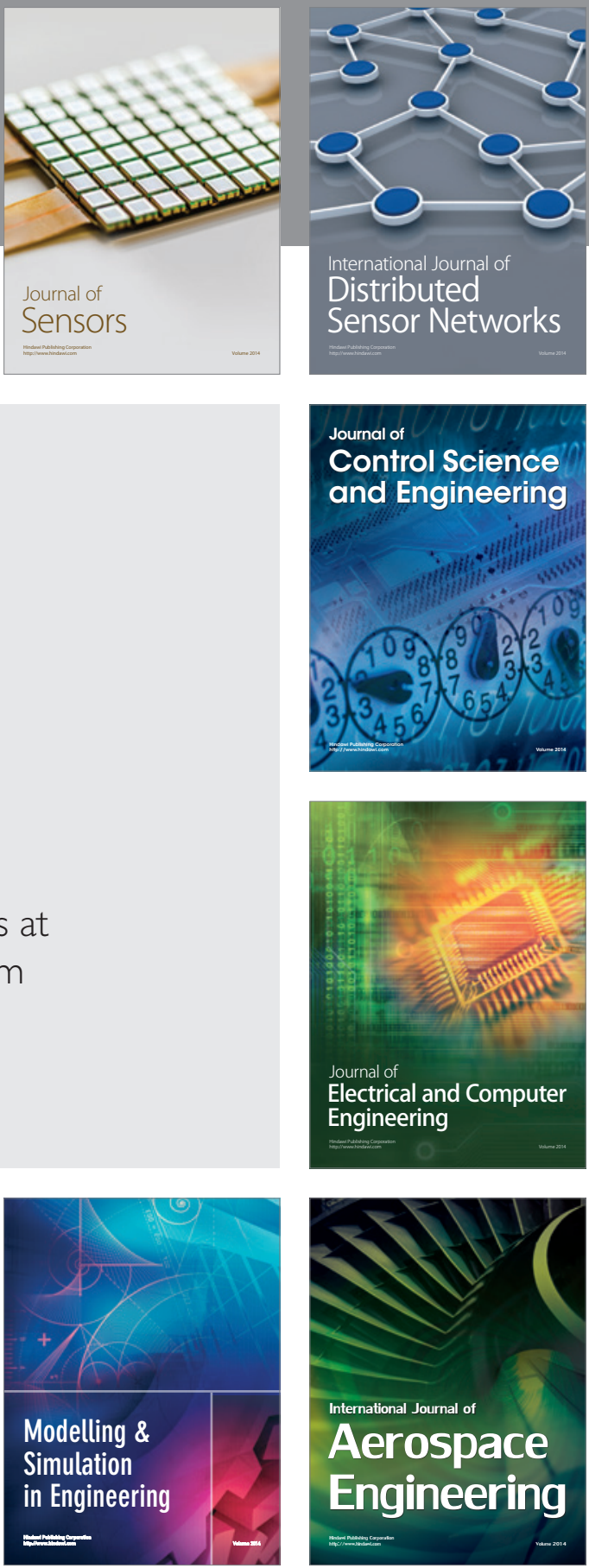

Journal of

Control Science

and Engineering
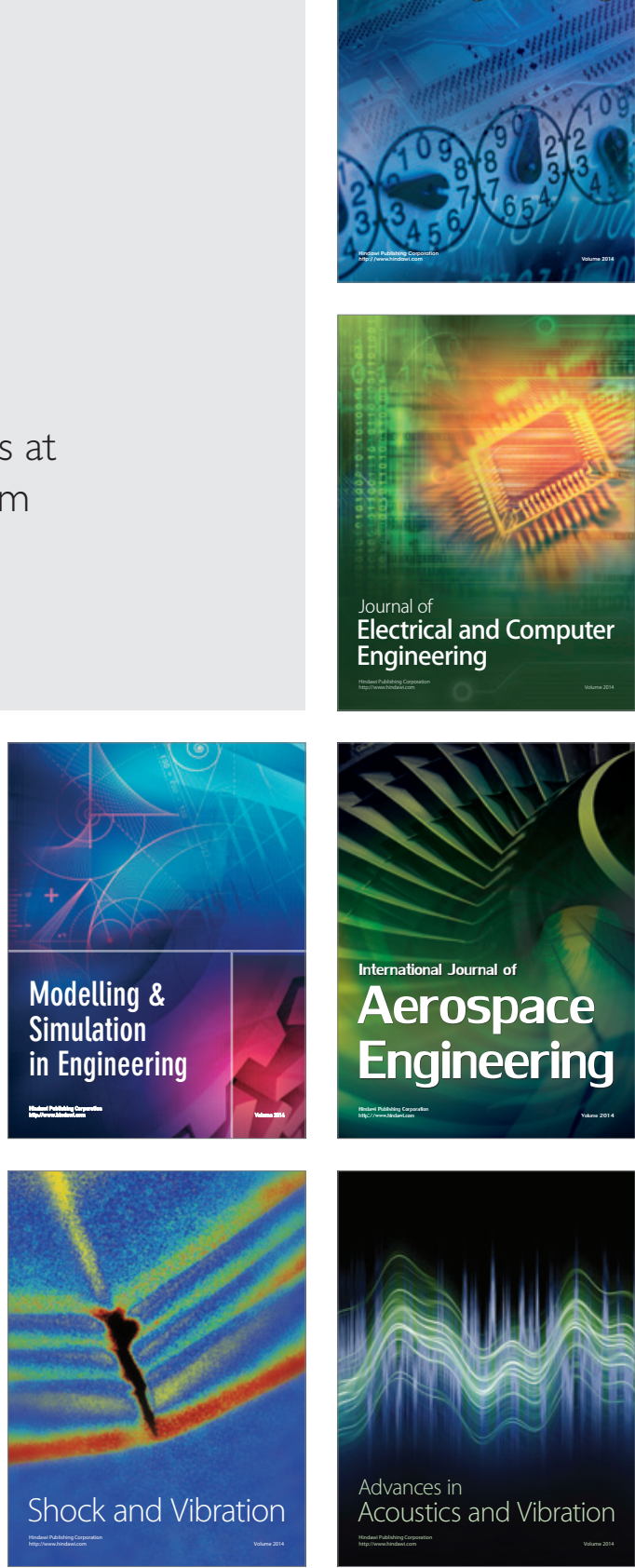